\title{
CRITERIOS PARA CONSTRUIR UNA COMPUTADORA PARA ESTUDIANTES DE LA CARRERA TECNOLOGIAS DE LA INFORMACION EN LA UNIVERSIDAD ESTATAL DEL SUR DE MANABI
}

\author{
AUTORES: Jose Efrain Alava Cruzatty ${ }^{1}$ \\ Magdalena Toala Zambrano \\ Victor Antonio Figueroa Castillo ${ }^{3}$ \\ Genesis Michelle Rodriguez Sinisterra ${ }^{4}$
}

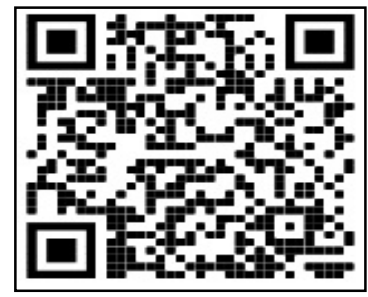

\section{DIRECCIÓN PARA CORRESPONDENCIA: jose.alava@unesum.edu.ec}

Fecha de recepción: 10/04/2020

Fecha de aceptación: 26/11/2020

\section{RESUMEN}

El presente trabajo se realizó con la finalidad de brindar información que ayude en la toma de decisiones al momento de construir un ordenador para estudiantes, para cubrir sus necesidades y que sirva de apoyo en su desenvolvimiento y rendimiento académico en el ámbito educativo. Los criterios para construir un ordenador se basan en conocer los componentes de los equipos microinformáticos y su factibilidad al momento de ejecutar tareas y procesos en los mismos, puesto que de acuerdo a la necesidad de cada usuario se pueden mejorar los recursos y el rendimiento de estos dispositivos. Se debe tomar en cuenta el uso que se le dará al equipo microinformático para así tomar en cuenta los aspectos más importantes entre los cuales tenemos el almacenamiento, la memoria, procesador y la autonomía. Al tomar en cuenta los criterios que se necesitan para construir un ordenador informático se tendrá una mayor comodidad para ejercer los diferentes procesos a realizar en el ámbito profesional. Lo más importante al momento de adquirir un dispositivo microinformático es que este cumpla con las necesidades del consumidor, ya que no está bien comprar un ordenador y desecharlo en pocos meses por que nos damos cuenta de que no nos resulta útil.

Palabras claves: Cambio tecnológico; Criterios; Dispositivos microinformático.

\footnotetext{
${ }^{1}$ Ingeniero en Telecomunicaciones y Máster en Telecomunicaciones. Personal académico auxiliar 1. Profesor, Miembro Técnico de la Dirección de Aseguramiento de la Calidad. Universidad Estatal del Sur de Manabí. Jipijapa, Manabí -Ecuador. jose.alava@unesum.edu.ec

${ }^{2}$ Ingeniera Civil y Máster en Seguridad y Salud Ocupacional. Personal académico auxiliar 1. Profesor. Universidad Estatal del Sur de Manabí. Jipijapa, Manabí - Ecuador. maria.toala@unesum.edu.ec

${ }^{3}$ Estudiante de la Carrera Tecnologías de la información. Bachillerato. Cuarto semestre. Estudiante de la Universidad Estatal del Sur de Manabí. Jipijapa, Manabí y Ecuador. Victor-fige@outlook.com

${ }^{4}$ Estudiante de la Carrera Tecnologías de la información. Bachillerato. Cuarto semestre. Estudiante de la Universidad Estatal del Sur de Manabí. Jipijapa, Manabí y Ecuador. genesiitha_rodriiguez@hotmail.com
} 
Jose E. Alava Cruzatty, Magdalena Toala Zambrano, Victor A. Figueroa Castillo, Genesis M. Rodriguez Sinisterra

\title{
CRITERIA FOR BUILDING A COMPUTER FOR CAREER STUDENTS INFORMATION TECHNOLOGIES AT THE SOUTHERN MANABI STATE UNIVERSITY
}

\begin{abstract}
The present work was carried out with the purpose of providing information that helps in making decisions when building a computer for students, to meet their needs and to support their development and academic performance in the field of education. The criteria for building a computer are based on knowing the components of microcomputer equipment and their feasibility when executing tasks and processes in them, since according to the needs of each user, resources and performance of these can be improved. dispositives. It should be taken into account the use that will be given to the microcomputer equipment to take into account the most important aspects among which we have storage, memory, processor and autonomy. When taking into account the criteria that are needed to build a computer will have a greater comfort to exercise different processes to perform in the professional field. The most important at the time of acquiring a microcomputer device is that it meets the needs of the consumer, since it is not good to buy a computer and discard it in a few months because we realize that it is not useful.
\end{abstract}

Keywords: Criteria; Microcomputer devices; Technological change.

\section{INTRODUCCIÓN}

Los avances tecnológicos en computación y software son constantes. Esto hace que frecuentemente exista la duda de cuándo renovar el computador, especialmente debido a la presión existente en los medios de comunicación y aparición de nuevos equipos y programas. La decisión generalmente se toma basada en elementos de carácter subjetivo, por lo que es importante realizar una evaluación de una manera apropiada. (Cosoi P., 2001)

El pronóstico en ventas de computadores en América Latina para el último trimestre del 2009 tuvo un aumento del 29,6\% en comparación con el mismo período del año 2008. Así lo estima la firma de inteligencia de mercados Gartner, basado en un incremento estimulado por el número de ventas de portátiles y netbooks. Resulta fundamental saber qué tipo de computador comprar, según las necesidades de cada usuario. Roberto Vélez, gerente de Marketing de AOC en Latinoamérica, opina que "la tendencia ahora es hacia un PC que pueda realizar trabajos básicos y cotidianos, que tenga un buen desempeño en el uso del procesador de texto, navegación en internet, correo electrónico, chat, así como el hecho de tener una buena experiencia en sonido, video o imagen”. (EL ESPECTADOR, 2009)

La presente investigación está enfrascada a ofrecer una visión precedente de lo que necesita tener un ordenador antes de ser comprado, para poder resolver las distintas necesidades del usuario, que exige de un equipo microinformático fiable y confiable para poder tener un trabajo optimo, de muy buen desempeño, con un rendimiento necesario para su área.

Según el artículo sobre el "Alcances y limitaciones de la noción de impacto social de la ciencia y la tecnología”, se presentan algunas aproximaciones a la noción de impacto social de la ciencia y la tecnología, acotando dimensiones con el propósito de definir de un modo operativo un 
fenómeno cuya medición es requerida para la evaluación de políticas en ciencia, tecnología e innovación. A tal fin, el texto se desarrolla en dos direcciones: una de naturaleza teórica y la otra más propiamente metodológica. En lo teórico se reseñan los principales problemas conceptuales que presenta la noción de impacto social, y se revisan algunas de las perspectivas existentes acerca de los modos y las dinámicas que adopta la producción y difusión del conocimiento científico y tecnológico. En cuanto a lo metodológico, el artículo expone algunas de las dimensiones sociales propuestas que debería contemplar el análisis de los procesos de impacto, y señala cuáles son los niveles de observación que admite un análisis de este tipo. (Albornoz, Estébanez, \& Alfaraz, 2005)

Es así que al pasar el tiempo, las necesidades de un consumidor van cambiando y cada vez más las actualizaciones de programas informáticos exigen más características físicas en un ordenador, por lo tanto los requerimientos físicos deben ser acordes a las exigencias y es más deben estar preparados para futuras actualizaciones de las distintas aplicaciones que se podrían utilizar, en concurrencia la presente investigación se basa en la actualidad, teniendo en cuenta el desarrollo de la tecnología, sobre todo el direccionamiento que está tomando hoy en día.

Finalmente al ver que la tecnología va avanzando y que los estudiantes informáticos tienen necesidades diferentes al resto de personas, hay que tomar en cuenta las características básicas que debe tener el dispositivo informático que a construir ya que normalmente no se adquiere el correcto, por lo tanto sabiendo la especificación de los dispositivos correctos se logra tener un ordenador con buen rendimiento que va a permitir realizar las actividades y procesos útiles que deseas con la fluidez que se necesitas.

\section{DESARROLLO}

En la actualidad muchos de los estudiantes universitarios desconocen cómo elegir un ordenador adecuado para cubrir sus necesidades, es así que no toman en cuenta el rendimiento que exigen ellos, lo cual conlleva a adquirir ordenadores poco factibles que retrasan su aprendizaje.

Por lo tanto, al impartir los conocimientos sobre la construcción de un ordenador a los estudiantes de la carrera Tecnologías de la Información y Comunicación en la Universidad Estatal del Sur de Manabí, se desarrolla la noción necesaria para adquirir un dispositivo microinformático.

Se busca desarrollar las habilidades que permitan elegir correctamente un ordenador para brindar mayor comodidad al momento de gestionar las tareas que se ejecuten por parte del estudiante.

Según (BENEFICIOS, s.f.) "Los individuos que viven en la sociedad naciente, difícilmente logran imaginar que hace medio siglo sus ascendencias eran prácticamente independiente de las tecnologías de redes informáticas mundiales.”

Desde la perspectiva de los informáticos y las personas hoy en día las tecnologías han evolucionado en gran parte a las necesidades de los problemas y aspectos básicos que tiene la humanidad, que prácticamente van surgiendo al pasar de los años según (Pueyo, 2016) "la tecnología es el factor que más se desarrolla al pasar de los años. Se desarrolla principalmente en la informática, robótica y la electrónica. Este desarrollo ha traído como consecuencia grandes aportes a la humanidad.” 
Jose E. Alava Cruzatty, Magdalena Toala Zambrano, Victor A. Figueroa Castillo, Genesis M. Rodriguez Sinisterra

La informática se ha distribuido progresivamente en todos los ámbitos profesionales donde claramente notamos los avances tecnológicos, que permiten la comunicación y el manejo de la información que hoy disfrutamos. Por lo tanto, la formación académica en los jóvenes es completa en todos los sentidos.

Para hablar del computador y sus componentes primero debemos conocer su definición y un poco de su historia. El computador es un dispositivo microinformatico que tiene como finalidad agilizar procesos, gestionar recursos, facilitar y organizar información que el usuario requiere, mediante procedimientos matemáticos y lógicos controlados por software los cuales permiten comunicación directa entre hombre - maquina a traves de sus perifericos.

\section{BREVE HISTORIA DE LOS COMPUTADORES.}

En 1670 el filósofo y matemático alemán Gottfried Wilhelm Leibniz perfeccionó la máquina de calcular de Pascal e inventó una que también podía multiplicar. (Castillo T., 2013). El inventor francés Joseph Marie Jacquard, al diseñar un telar automático, utilizó delgadas placas de madera perforadas para controlar el tejido utilizado en los diseños complejos. Durante la década de 1880 el estadístico estadounidense Herman Hollerith concibió la idea de utilizar tarjetas perforadas, similares a las placas de Jacquard, para procesar datos. Hollerith consiguió compilar la información estadística destinada al censo de población de 1890 de Estados Unidos mediante la utilización de un sistema que hacía pasar tarjetas perforadas sobre contactos eléctricos. (Castillo T., 2013)

También en el siglo XIX el matemático e inventor británico Charles Babbage elaboró los principios de la computadora digital moderna. Inventó una serie de máquinas, como la máquina diferencial, diseñadas para solucionar problemas matemáticos complejos. Muchos historiadores consideran a Babbage y a su socia, la matemática británica Augusta Ada Byron (1815-1852), hija del poeta inglés Lord Byron, como a los verdaderos inventores de la computadora digital moderna. La tecnología de aquella época no era capaz de trasladar a la práctica sus acertados conceptos; pero una de sus invenciones, la máquina analítica, ya tenía muchas de las características de un ordenador moderno. Incluía una corriente, o flujo de entrada en forma de paquete de tarjetas perforadas, una memoria para guardar los datos, un procesador para las operaciones matemáticas y una impresora para hacer permanente el registro. (Castillo T., 2013)

Los ordenadores analógicos comenzaron a construirse a principios del siglo XX. Los primeros modelos realizaban los cálculos mediante ejes y engranajes giratorios. Con estas máquinas se evaluaban las aproximaciones numéricas de ecuaciones demasiado difíciles como para poder ser resueltas mediante otros métodos. Durante las dos guerras mundiales se utilizaron sistemas informáticos analógicos, primero mecánicos y más tarde eléctricos, para predecir la trayectoria de los torpedos en los submarinos y para el manejo a distancia de las bombas en la aviación. (Castillo T., 2013) 
Durante la II Guerra Mundial (1939-1945), un equipo de científicos y matemáticos que trabajaban en Bletchley Park, al norte de Londres, crearon lo que se consideró el primer ordenador digital totalmente electrónico: el Colossus. Hacia diciembre de 1943 el Colossus, que incorporaba 1.500 válvulas o tubos de vacío, era ya operativo. Fue utilizado por el equipo dirigido por Alan Turing para descodificar los mensajes de radio cifrados de los alemanes. En 1939 y con independencia de este proyecto, John Atanasoff y Clifford Berry ya habían construido un prototipo de máquina electrónica en el Iowa State College (EEUU). Este prototipo y las investigaciones posteriores se realizaron en el anonimato, y más tarde quedaron eclipsadas por el desarrollo del Calculador e integrador numérico electrónico (en inglés ENIAC, Electronic Numerical Integrator and Computer) en 1946. El ENIAC, que según se demostró se basaba en gran medida en el ordenador Atanasoff-Berry (en inglés ABC, Atanasoff-Berry Computer), obtuvo una patente que caducó en 1973, varias décadas más tarde. (Castillo T., 2013)

El ENIAC contenía 18.000 válvulas de vacío y tenía una velocidad de varios cientos de multiplicaciones por minuto, pero su programa estaba conectado al procesador y debía ser modificado manualmente. Se construyó un sucesor del ENIAC con un almacenamiento de programa que estaba basado en los conceptos del matemático húngaro-estadounidense John von Neumann. Las instrucciones se almacenaban dentro de una llamada memoria, lo que liberaba al ordenador de las limitaciones de velocidad del lector de cinta de papel durante la ejecución y permitía resolver problemas sin necesidad de volver a conectarse al ordenador. (Castillo T., 2013)

A finales de la década de 1950 el uso del transistor en los ordenadores marcó el advenimiento de elementos lógicos más pequeños, rápidos y versátiles de lo que permitían las máquinas con válvulas. Como los transistores utilizan mucha menos energía y tienen una vida útil más prolongada, a su desarrollo se debió el nacimiento de máquinas más perfeccionadas, que fueron llamadas ordenadores o computadoras de segunda generación. Los componentes se hicieron más pequeños, así como los espacios entre ellos, por lo que la fabricación del sistema resultaba más barata. (Castillo T., 2013)

A finales de la década de 1960 apareció el circuito integrado (CI), que posibilitó la fabricación de varios transistores en un único sustrato de silicio en el que los cables de interconexión iban soldados. El circuito integrado permitió una posterior reducción del precio, el tamaño y los porcentajes de error. El microprocesador se convirtió en una realidad a mediados de la década de 1970, con la introducción del circuito de integración a gran escala (LSI, acrónimo de Large Scale Integrated) y, más tarde, con el circuito de integración a mayor escala (VLSI, acrónimo de Very Large Scale Integrated), con varios miles de transistores interconectados soldados sobre un único sustrato de silicio. (Castillo T., 2013) 
A finales de siglo XX surgiron los microcircuitos con una alta integracion, funcionando ademas con Paralelismo y emulando algunas características de las redes neurales con las que funciona el cerebro humano. Como lo es la Inteligencia Artificial, Interconexión entre todo tipo de computadoras, dispositivos y redes (redes integradas), además de la integración de datos, imágenes y voz (entorno multimedia). (Delfin, 2013)

Con la utilización del lenguaje natural (lenguaje de quinta generación). Estos conceptos merecen una somera explicación, debido a que si representan avances cualitativos con respecto a las generaciones anteriores. La mayoría de las computadoras actuales ejecutan las instrucciones del lenguaje de maquina en forma secuencial, es decir, efectúan una sola operación a la vez. Sin embargo, en principio también es posible que una computadora disponga de varios procesadores centrales, y que entre ellos realicen en forma paralela varias operaciones, siempre y cuando estas sean independientes entre sí.(Delfin, 2013)

\section{LAS COMPUTADORAS EN LA ACTUALIDAD}

Desde la innovación misma de los sistemas basados en microprocesadores y la gran demanda que tienen las empresas dedicada en este sector tecnológico por este tipo de artículos, es difícil predecir el camino que seguirán estos dispositivos. Es por ello que continuamente las numerosas compañías están innovando nuevos productos para poder obtener el éxito esperado y competir con las grandes empresas que se dedican a presentar productos similares. En otras palabras, la publicidad y el diseño juegan un papel muy importante para lograr el auge esperado. (Berral Montero, 2014)

La fabricación y diseño por el que están atravesando los sistemas programables es provocada por la constante evolución de los sistemas y equipos que depende es este. (Montero, 2010)

Sin embargo, se pueden delimitar cuatro grandes tendencias en los sistemas digitales programables:

1. Los grandes ordenadores o mainframes. Se centran en lograr sistemas para procesado masivo de datos en el menor tiempo posible. Su costo es muy elevado y se dedican a aplicaciones de investigación, gestión de grandes cantidades de datos, etc. (Montero, 2016)

2. Los ordenadores personales y las estaciones de trabajo. El propósito de este tipo de equipos es aumentar la productividad individual, dando la mayor capacidad posible a cada puesto de trabajo. (Berral Montero, 2014) 
La competencia entre distintos fabricantes y el abaratamiento de los precios en esta parcela han colaborado a llevar este tipo de equipos a un entorno doméstico, de uso particular. (Berral Montero, 2014)

3. Tabletas y Smartphone o teléfono inteligentes. Este tipo de dispositivos hoy en día está cada vez más en el mercado de uso personal y profesional, de tal forma que es parte fundamental en el día a día de cada uno de nosotros por las diversas funcionalidades que se inculcaron y que hoy están ayudando a gran parte de la sociedad. (Henry Bautista, 2018)

4. El control industrial y los automatismos. Según (Montero, Equipos Microinformáticos, 2016, pág. 2) “Se aprovecha la potencia de los microcontroladores en prácticamente cualquier sistema electrónico para lograr la automatización a gran escala en nuestra sociedad"

Debido a esto es que en la actualidad se están realizando cada vez más microprocesadores con menor tamaño y mayor velocidad para poder obtener mayor efectividad en las funciones que realiza. (Montero, 2010)

Es así que notamos que en este grupo también se involucran los ordenadores personales y actualmente también podemos involucrar a los Smartphone por lo que están dedicados en parte al estudio. (Montero, 2010)

Un microcontrolador es un microprocesador con memoria y puertos de comunicaciones integrados en el chip. (Berral Montero, 2014)

La estructura básica de un sistema basado en microprocesador se muestra en la Figura 1.1, en la que podemos observar los distintos elementos de que consta: el procesador, la memoria y las unidades de entrada y salida, todos ellos interconectados a través de tres buses:

\section{$\checkmark$ Direcciones \\ $\checkmark$ Datos \\ $\checkmark$ Control. (Berral Montero, 2014)}

Un bus es un conjunto de cables o hilos conductores que entre todos transportan una información. . (Montero, Operaciones auxiliares de montaje de componentes informáticos, 2010, págs. 2,3)

Las unidades de entrada y salida permiten que el equipo se relaciones con el mundo exterior: teclado, ratón, disco duro, lector de DVD, impresora, monitor, escáner, sondas de temperatura, cámaras de vídeo, etc. . (Montero, Operaciones auxiliares de montaje de componentes informáticos, 2010, págs. 2,3) 
El funcionamiento básico de este sistema programable es bastante sencillo; en la memoria se almacena un programa que va ejecutando el procesador al ritmo marcado por el reloj y datos con los que trabajará; podemos decir por tanto que el procesador es el cerebro del equipo, se encarga de ir ejecutando cada instrucción u orden que aparece en la memoria, cosas como leer un dato de una unidad de entrada, operaciones aritméticas, operaciones lógicas, etc. A partir de este funcionamiento básico, y mediante un programa (que se denomina software), se consigue hacer todo lo que hoy en día conocemos de los ordenadores y por supuesto muchas más cosas que van surgiendo día a día. . (Montero, Operaciones auxiliares de montaje de componentes informáticos, 2010, págs. 2,3)

Sin embargo, este diagrama de los bloques no es aplicable directamente a lo que actualmente es un Ordenador Personal. En un ordenador personal tenemos dispositivos controladores de acceso directo a memoria, controladores de disco duro, de teclado, procesadores gráficos, memoria RAM en varios niveles de jerarquía, etc., de tal forma que para un equipo microinformático basado en PC (Personal Computer), su estructura es más compleja, desde el punto de vista del personal técnico de mantenimiento o reparación, teniendo en cuenta las que denominaremos unidades mínimas reparables actualmente. . (Montero, Operaciones auxiliares de montaje de componentes informáticos, 2010, págs. 2,3)

Por “unidad mínima reparable” se entenderá la parte hardware del equipo que se sustituirá en caso de avería, ya que no compensa la sustitución de un componente determinado de la tarjeta estropeada por cuestiones de tiempo de reparación (costo de mano de obra) y equipo de diagnóstico necesario; resulta más caro cambiar un componente de una tarjeta que adquirir una tarjeta nueva y cambiarla. (Montero, Operaciones auxiliares de montaje de componentes informáticos, 2010, págs. 2,3)

\section{Estructura externa de un equipo microinformático}

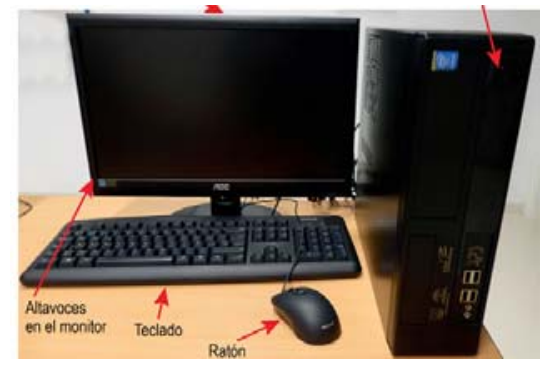

GRAFICO N.1 Estrutura externa de un equipo microinformatico. 
La estructura externa de un equipo microinformático basado en PC puede decirse que actualmente consta de los siguientes elementos, que se muestran En el GRAFICO N.1. (Blogia, 2004)

En la Unidad Central (CPU), se ubican las unidades de almacenamiento de información (disquetera, disco duro, lector de tarjetas...), lector de CD-ROM, tarjeta gráfica, tarjeta de sonido, etc., además del microprocesador y de la memoria. (Blogia, 2004)

Los elementos externos a la unidad central se conectan mediante sus respectivos cables con conector en su extremo y que son distintos para cada elemento, aunque hay elementos en que el tipo de conector es el mismo. Un ejemplo de los conectores en una unidad central se muestra en el GRAFICO N.2(Blogia, 2004)

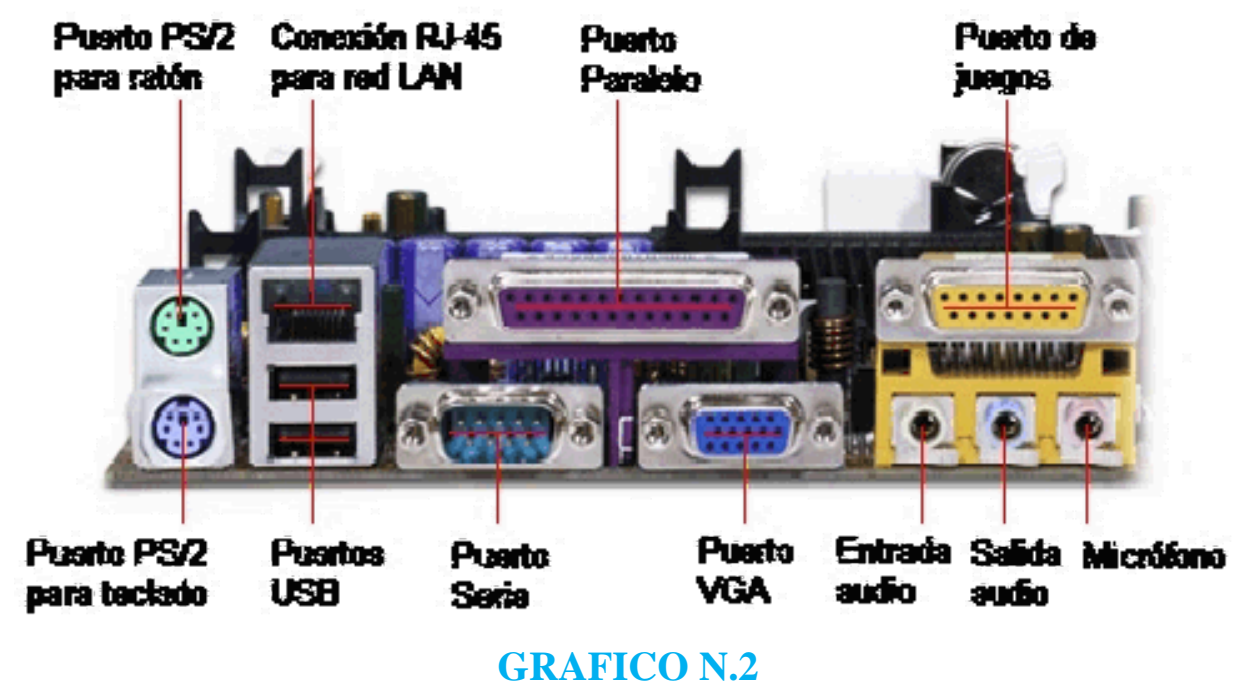

Conectores normalizados utilizados en una unidad central de PC

El conector PS/2 hembra de color verde es el del ratón.

El conector PS/2 hembra de color violeta es el del teclado.

Estos conectores son los siguientes:

> DB-9 macho: conector de 9 patillas. Es el conector serie RS-232C. Se designa como COM1.

$>$ DB-25 macho: conector de 25 patillas. Es el conector serie RS-232C. También puede ser de 9 patillas. Se designa como COM2. 
DB-25 hembra: conector de 25 agujeros. Es el conector paralelo centronics. Se designa como LPT1.

$>$ DB-15 HD (alta densidad) hembra: salida de video 15 agujeros. Es el conector de salida de la tarjeta gráfica VGA y SVGA.

$>$ DB-15 hembra: es el conector de juegos (joystick).

> PS/2 hembra: es el conector del teclado y ratón tipo PS/2, también denominado miniDIN.

$>$ USB: es el conector del bus serie universal.

$>$ DIN hembra: es de 5 agujeros y corresponde al conector del teclado estándar.

$>$ R1-11: conector de módem para enganchar a un teléfono y a la línea telefónica (en caso de módem interno). (Blogia, 2004)

\section{Estructura físico-funcional de un equipo microinformático}

Cuando hablamos de la estructura físico-funcional nos referimos a una estructura física real, en la que además cada elemento cumple su función y se suele encontrar por separado suministrado por distintos fabricantes. En ella se puede ver la unidad central que lleva asociados los elementos de entrada (en el lado izquierdo), los de salida (en el lado derecho) y los internos (dentro y debajo): obsérvese que, en los dispositivos de salida, el elemento OTROS es bidireccionales. Debido a que existen en el mercado muchos dispositivos que se pueden conectar al puerto paralelo y serie para entrada y salidas de datos. Se han representado solo algunos de los dispositivos más comunes para mayor claridad. (Berral Montero, 2014).

La unidad central, es lo que denominamos CPU (Unidad Central de Proceso) o torre en el lenguaje coloquial, está formado por la carcasa, en cuyo interior se encuentra en la fuente de alimentación y la placa base (también incluye discos duros y unidades de CD/DVD o Bluray). (Berral Montero, 2014).

Según (Berral Montero, 2014) “La placa base incorpora los zócalos para el procesador y la memoria, los conectores para el resto de elementos del equipo informático. Como discos duros, lectores de tarjetas, tarjeta gráfica, etc.”

Luego de instruirse un poco en el mundo de los dispositivos microinformáticos y saber un poco de su historia, evolución y su importancia es hora de entrase en la parte importante de este artículo, el cual es reconocer los criterios a tomar en cuenta para obtener una computadora estudiantil que cumpla con las necesidades requeridas por el usuario. 
Lo que hay que saber primera mente, es que las partes que hay que tomar en cuenta siempre a la hora de obtener un ordenador son: Placa base, Memoria RAM, Almacenamiento, Procesador, Fuente de poder, tarjeta de video y refrigeración.

\section{Placa base}

Lo primero que hay que elegir al momento de armar un computador es la placa base, por lo que aquí se determina qué clase de ordenador vas a adquirir y se tomaran en cuenta los siguientes factores:

El tamaño de la placa base.

Debemos de saber que existen 3 formatos de tamaño entre los cuales tenemos el completo (ATX), tamaño medio (micro ATX) o tamaño reducido (mini ITX), las diferencia entre cada formato son la cantidad de ranuras, conectores y facilidad de expansión de se tendrá a futuro. (Ruben Andres, 2016)

\section{El Zócalo.}

Hay que saber que el zócalo es el lugar donde va ubicado el procesador, por lo tanto, es preferible elegir la placa base con el zócalo adecuado para el procesador que tenemos en mente y que vamos a adquirir.

Debemos de conocer que actualmente Intel utiliza el zócalo LGA 1151 para sus procesadores domésticos, el zócalo LGA 2011 (o su variante LGA 2011-3) para sus gamas de muy alto rendimiento y procesadores para servidores. Por su parte, AMD utiliza zócalos FM2 y FM2+ para sus procesadores con chip gráfico integrado y el zócalo AM3 y AM3+ para el resto de procesadores domésticos. (Ruben Andres, 2016)

Calidad de los circuitos.

Como todos sabemos en el mercado existen diferentes tipos de marcas, precios y calidad de funcionamiento en función al automatismo que se va a generar.

Por lo tanto al saber que tipo de trabajo vas a relizar con tu computador según (Ruben Andres, 2016) "es necesario tomar en cuenta la cantidad de metales conductores como el cobre o el oro en la fabricación de la placa base, contar con condensadores sólidos (mal llamados capacitores) y con disipadores de aluminio que permitan la refrigeración pasiva de los chips integrados en la placa." 
Los chip de la placa base.

Por lo general se deben tomar muy en cuenta esta parte porque esto determinara la cantidad y el tipo de conexiones que tendras al momento de quere coneciones SATA, PCIe, USB, netre otros. (Ruben Andres, 2016)

\section{Procesador.}

El procesador es parte fundamental del computador, se podría decir que el más importante de los componentes de una computadora, por lo que gracias a este dispositivo se puede procesar e interpretar la información y mantener comunicación entre maquina hombre. (Ruben Andres, 2016)

Por lo tanto, hay que tomar en cuenta vara de sus características.

Marca del procesador.

Como sabemos en el mercado las dos marcas más conocida de procesadores son Intel y ADM donde claramente estas dos empresas están luchando por liderar este mercado. (Ruben Andres, 2016)

La diferencia de calidad de procesamiento es notable por lo que las personas actualmente están optando por Intel, pero las desventajas de estos son su precio, por el contrario, con AMD sus precios son más cómodos, los cuales son menores a los de su competencia directa, pero esto no quiere decir que no sería factible comprar un procesador de esta marca, esto ya depende más del usuario y su presupuesto. (Ruben Andres, 2016)

Núcleos, hilos y frecuencia.

Al elegir un procesador lo primero que deberías de ver son sus núcleos, los cuales permiten un procesamiento independiente y un mayor rendimiento a la hora de realizar tareas, por otra parte, hay que tomar en cuenta los hilo que tiene cada nucleó sobre todo porque permitiendo duplicar las capacidades de trabajo del procesador, obteniendo un mejor rendimiento, lo cual es un factor que se necesita en informática. (Ruben Andres, 2016)

Según (Ruben Andres, 2016) "La tecnología que permite utilizar más de un hilo por núcleo se conoce como HyperThreading e Intel lleva más de una década usándola en sus productos.” Por otro lado otro factor a tomar muy en cuenta es la frecuencia con la que trabajan los procesadores lo cual se expresa en Megahercios(MHz), esto permite tambien ver el rencimiento con el que 
trabajan los nucleós por ellos es fundamentan saber esto a la hora de adquirir un procesador. (Ruben Andres, 2016)

La memoria cache.

Este es uno de los criterios que siempre se pasa por alto a la hora de comprar un procesador. Por consiguiente hay que saber que esto puede marcar una gran diferencia en el rendimineto puesto que a mayor memoria cache, mas cantidad de datos pueden estar dicpuestos para optimizar el funcionamiento del chip. (Ruben Andres, 2016)

\section{Consumo Energetico.}

Hay que sarber que el consumo de electricidad varia entorno a los diferentes procesadores por lo que a mayor potencia mayor consumo eléctrico.

No solamente es importante tener en cuenta el consumo electrico sino que esto tambien implica la cantidad de energia que vas a utilizar con este procesador, lo que tambien afecta al aumento de temperatura del procesador y sus chip integrados lo que va a requerir un enfrianmento adecuador para que no falle el procesador. (Ruben Andres, 2016)

\section{Almacenamiento}

Primeramente, hay que saber que actualmente existen 3 tipos de disco de almacenamiento entre los cuales tenemos el HHD, SSH y los discos híbridos los cuales es una unión de los 2 primeros.

Los discos HHD son los que naturalmente toda persona promedio poseen en un ordenador por lo que no tiene un coste elevado para adquirir, por lo general actualmente un disco de $1 \mathrm{~T}$ ronda entre los 100 y 150 dólares, con lo que se obtiene un gran almacenamiento por poco dinero, uno problema de este tipo de disco es que al momento de una caída o al mojarse es muy probable perder la información del usuario que conlleva este se pierda y no se pueda recuperar por lo que las pista y sectores esta dañados y es más estos son muy susceptibles a daños mecánicos

Por consiguiente, los discos SSD o también conocidos como discos solidos son un tipo de disco con memoria solida es decir se asemejas a una memoria flash, lo que permite tener hasta el triple de velocidad que los discos HHD y, es más también son muy silenciosos con un menor consumo de energía, mayor velocidad de transición de datos y resistentes a choques, su grande desventaja es que su adquisición no es muy barata.

Los factores que se deben tomar en cuenta para escoger un dispositivo de almacenamiento es la velocidad, el almacenamiento y su tipo de conexión 
Es este caso como un estudiante de informática a la larga de tu carrera tendrás que guardar y almacenar gran cantidad de información por lo que es recomendable por lo menos adquirir 1T de almacenamiento lo cual lo puedes obtener con un disco HHD, pero también deseas velocidad es así que puede conectar también un disco SSD el cual lo puede adquirí si tu presupuesto lo puede cubrir, como son muy caros esto disco, es recomendable solo comprar un SSD de 125Gb, en el cual puedas instalar aquí el sistema operativo y tus programa para así tener mayor velocidad al ejecutar procesos y tendría para almacenamiento de datos el disco de 1T.

\section{Memoria RAM.}

La memoria RAM (random access memory) o memoria de acceso aleatorio es un tipo de memoria volátil que permite almacenar datos temporalmente. (Ruben Andres, 2016)

La memoria RAM aportará fluidez a tu sistema, por lo que la cantidad a instalar y el tipo de memoria dependerá mucho del uso que vayas a hacer del ordenador, capacidad de la placa elegida e incluso el sistema de refrigeración que tengas instalado en tu PC,los parámetros que hay que tomar en cuenta son los siguientes. (Ruben Andres, 2016)

Cantidad de memoria RAM.

La cantidad de memoria estará directamente relacionada con el uso que hagas del equipo. Cada uso requiere una cantidad de RAM diferente. No necesitas la misma memoria RAM para navegar o ver películas en tu ordenador, que para jugar o editar vídeo o fotos. (Ruben Andres, 2016)

Como norma general, un PC para uso doméstico tendrá suficiente con 4 u 8 GB de memoria RAM, mientras que un PC para juegos necesitará entre 8 y 16 GB y una estación de trabajo que se utilice para diseño, edición de vídeo o fotografía podría necesitar entre 16 y 32 GB de memoria RAM. (Ruben Andres, 2016)

Tamaño y formato

Actualmente, la gran mayoría de las placas base utiliza el tipo de memoria DDR3, pero los fabricantes ya comienzan a lanzar nuevos modelos que soportan el nuevo formato DDR4. (Ruben Andres, 2016)

Los distintos formatos de memoria RAM (DDR400, DDR2, DDR3 y DDR4) se diferencian por el número de contactos del conector de la placa base. Además, cada tipo cuenta con diferentes muescas en el conector que impide que, por ejemplo, puedas confundirlos e intentar conectar un módulo DDR2 en un conector DDR3. Algo más habitual de lo que podría llegarse a pensar. (Ruben Andres, 2016) 
Como hemos comentado, estamos en plena transición entre la memoria DDR3 y DDR4, por lo que debes asegurarte de elegir la memoria RAM adecuada para tu placa base. (Ruben Andres, 2016)

Algunos módulos incorporan disipadores de aluminio que mejoran la refrigeración de los chips de memoria, pero también elevan su altura y pueden llegar a tocar con el disipador que refrigera el procesador. (Ruben Andres, 2016)

Por lo tanto, a la hora de elegir los módulos de memoria, ten en cuenta las medidas de ambos elementos ya que unas memorias demasiado altas podrían impedir la instalación de un disipador sobredimensionado para el procesador.

Latencias en la RAM.

Junto a la frecuencia, otro de los parámetros que tienes que tener en cuenta a la hora de elegir la memoria RAM adecuada para tu ordenador son las latencias de la memoria. (Ruben Andres, 2016)

La estructura interna de la memoria RAM es como la de un tablero de ajedrez tridimensional en el que cada cuadro del tablero es una celda en la que se escriben los datos que se almacenan. (Ruben Andres, 2016)

La latencia es el tiempo que tarda la memoria RAM en situarse en una determinada celda para leer o escribir su contenido. Cuanto mayor sea la latencia de la memoria RAM, mayor es el tiempo que "pierde" en llegar a una determinada celda y, por lo tanto, menos eficiente en su trabajo. (Ruben Andres, 2016)

El parámetro de latencia en los módulos acostumbra a mostrarse tras las siglas CL (CAS Latency) que expresa el valor de la latencia CAS de la memoria, que a su vez es uno de los valores más importantes a la hora de establecer la eficiencia de la memoria RAM. (Ruben Andres, 2016)

Por lo tanto, a igualdad de frecuencias de reloj para un módulo de memoria RAM, es preferible elegir una memoria RAM con una latencia baja. Por ejemplo, un módulo con una latencia CL7 es más eficiente que uno con una latencia CL8, CL9 o CL10. (Ruben Andres, 2016) 
El voltaje.

Si te damos a elegir entre un coche que consuma mucho combustible y un coche que consuma muy poco, probablemente no tendrás muchas dudas al elegir el que menos consume, para de ese modo ahorrar algo de dinero en gasolina. (Ruben Andres, 2016)

Pues en la memoria RAM sucede algo similar. Aunque la mayoría de los módulos DDR3 disponibles están diseñados para consumir un máximo de 1,5 voltios, existen algunos que rebajan esa cuota de consumo y la sitúan en torno a los 1,35 voltios.

Estos consumos, en situaciones normales simplemente implican un menor consumo energético. (Ruben, 2016)

\section{Fuente de poder}

La fuente de poder es un trasformador pequeño, el cual sirve para transformar energía Alterna en continua y aportar los voltios necesarios que necesita cada dispositivo de un computador.

Lo que se debe tomar en cuenta primeramente es:

Certificados.

Lo primero que se debe hacer es verificar los certificados existentes para conocer cuál es el mejor a elegir. (Hernadez, 2016)

Previamente les mencionamos que las fuentes de poder certificadas están clasificadas en base a la calidad de los componentes y la eficiencia que otorga cada uno de ellos. Dicha eficiencia debe ser obtenida en el 20\%, 50\% y 100\% de carga de la fuente y un factor de potencia de 0.9 o mayor al $100 \%$ de su carga. Esto significa que, si una fuente tiene una certificación 80 Plus, el $80 \%$ o más de la energía tomada del circuito eléctrico es usada como energía para nuestro PC y el $20 \%$ restante de la energía consumida se pierde transformada en calor generado por la fuente de poder, por ello a continuación les describiremos las diferentes certificaciones que existen: (Hernadez, 2016)

- $\quad 80$ PLUS: Esta es la certificación más básica que podremos encontrar en una fuente de poder, prometiendo una eficiencia mínima del $80 \%$ en su funcionamiento general, por lo cual si tu presupuesto es el mínimo te recomendamos este tipo de fuentes en lugar de una fuente genérica para proteger tu equipo. Los modelos con esta certificación van desde los \$500 hasta $\$ 1,500$ pesos. (Hernadez, 2016) 
- $\quad 80$ PLUS Bronze: Esta es la certificación más popular en las fuentes de alimentación, por ello encontremos muchos modelos que gocen del certificado "Bronce" que es significado de que obtendremos hasta un 85\% de eficiencia máximo.. (Hernadez, 2016)

- $\quad 80$ PLUS Silver: Si tu presupuesto es más amplio podrás adquirir una fuente de poder con certificado "Plata", cuyos componentes son de mucha mejor calidad y promete una eficiencia de hasta $88 \%$ en un escenario del $50 \%$ de carga nominal. No obstante, es poco usual encontrar fuentes con este certificado en varios países, incluido México. (Hernadez, 2016)

- $\quad 80$ PLUS Gold: Esta certificación es utilizada en las fuentes de poder con cargas a partir de 550 watts en adelante, prometiendo ofrecer $90 \%$ de eficiencia durante su uso, una excelente característica para aprovechar al máximo la energía. Los precios de estos modelos van desde los $\$ 2,000$ hasta $\$ 5,500$ pesos. (Hernadez, 2016)

- $\quad 80$ PLUS Platinum: Esta es la certificación elite más popular que existe en el mercado de fuentes de alimentación, pues prometen un 92\% de eficiencia y están disponibles solamente en modelos desde 750 watts y con precios algo elevados para la mayoría de bolsillos que van desde $\$ 3,500$ hasta $\$ 7,000$ pesos. (Hernadez, 2016)

- $\quad 80$ PLUS Titanum: Esta es la mejor certificación que podremos encontrar en una fuente de alimentación, siendo implementada en 2011 para los equipos y usuarios más exigentes, pues este certificado está mayormente disponible en modelos con capacidades de 1,200 watts o más, por lo tanto, sus precios serán exageradamente altos que oscilan entre los \$6,000 y $\$ 10,000$ pesos, pero a cambio tendremos una eficiencia de hasta el 94\%.(Hernadez, 2016)

Fuente modular o no modular.

Esta es otra de las preguntas que usualmente tienen los usuarios, pues sabemos que entre las fuentes de poder certificadas existen modulares o no modulares, surgiendo la duda sobre qué modelo elegir. Sin embargo, en gustos se rompen géneros y sencillamente la decisión dependerá del usuario mismo, pues en base a las necesidades del mismo será el modelo que deberán elegir. (Hernadez, 2016)

- $\quad$ Modular: Ofrece diferentes conectores (varía según capacidad) para conectar diferentes periféricos con conectores ATX, EPS, SATA, PCIe y disquete, contando con la posibilidad de intercambiar los conectores. (Hernadez, 2016)

- $\quad$ No modular: Cuenta únicamente con un número definido de cables de conexión para utilizar sin la oportunidad de intercambiar entre sí. (Hernadez, 2016)

Siempre es preferible tener una fuente de poder modular para intercambiar los cables y conectores, según nuestras necesidades para no tener que utilizar molestos adaptadores, pero 
su precio es más elevado que los modelos ‘no modulares’. Así que la decisión de optar o no por un modelo modular quedará completamente en tus manos y tu bolsillo, pero si deseas una recomendación, opta por un modelo modular. (Hernadez, 2016)

\section{Case y Sistema de refrigeración.}

El tamaño adecuado

Uno de los factores más importantes a la hora de elegir una caja para tu PC, y dejando de lado el puramente estético, es el tamaño o factor de forma. (Ruben, COMPUTER HOY, 2016)

Éste factor de forma tiene que ser compatible con el formato de la placa base que has elegido, y contar con el espacio suficiente para los componentes que instalarás en ella. De especial relevancia será la tarjeta gráfica elegida, ya que puedes encontrarte que es demasiado grande para instalarla en el interior de la caja. (Ruben, COMPUTER HOY, 2016)

Además, también debe tener el ancho suficiente para permitir la instalación de los elementos opcionales de refrigeración del procesador que te mostraremos un poco más adelante. (Ruben, COMPUTER HOY, 2016)

\section{Materiales de fabricación}

Elegir una caja construida en aluminio o en acero reducirá las vibraciones de los componentes que forman tu PC y amortiguará el ruido de los sistemas de refrigeración interior, haciendo más confortable y silencioso el uso de tu ordenador. (Ruben, COMPUTER HOY, 2016)

\section{Capacidad de refrigeración}

La caja de tu PC actúa como soporte para todos los componentes de tu ordenador. Por lo tanto, debe estar diseñada para facilitar la instalación de los ventiladores adicionales que permitan la correcta refrigeración del interior de tu equipo. (Ruben, COMPUTER HOY, 2016)

Sistemas de gestión de cables

Puede parecer secundario, pero los sistemas internos de gestión de cables también son importantes. (Ruben, COMPUTER HOY, 2016)

Estos sistemas internos de gestión de cables consisten en una serie de orificios, ranuras y grapas que ayudan a canalizar el cableado a lo largo de la distribución interna del equipo, de forma que no entorpezca el paso del aire fresco por el interior de la caja. (Ruben, COMPUTER HOY, 2016) 
Esto también cumple con una clara función estética y de comodidad de trabajo en el interior de la caja ya que todo estará más ordenado y pulido y te resultará más sencillo conectar o desconectar los componentes si los cables están organizados, que si te encuentras con una maraña de cables sin principio ni fin. (Ruben, COMPUTER HOY, 2016)

Sistemas anti polvo

El polvo es un enemigo silencioso que, si no pones medios, terminará por estropear tu equipo.

Muchas cajas para PC actuales cuentan con filtros antipolvo extraíbles en sus rejillas de ventilación. Estos filtros frenarán considerablemente la acción del polvo en el interior de tu PC, pero será necesario mantenerlos limpios para que no bloqueen la expulsión del aire caliente del interior. Estos filtros extraíbles facilitarán su limpieza y mantenimiento periódico. (Ruben, COMPUTER HOY, 2016)

Para la elaboración del artículo se utilizaron métodos de la Investigación Científica tales como métodos teóricos, empírico, estadísticos-matemáticos y población y muestras, los cuales ayudaron a la construcción del articulo y a la comprobación de los datos estadísticos obtenido por medio de las encuestas realizada a los estudiantes de la carrera Tecnologías de la Información de la Universidad Estatal de Sur de Manabí.

La presente investigación tiene como finalidad brindar mayor seguridad al elegir un dispositivo microinformático de acuerdo a las necesidades del usuario, entre las cuales tenemos el rendimiento, la autonomía, la fiabilidad y portabilidad del ordenador sin ninguna adversidad.

El impacto del presente artículo es lograr que todos y cada uno de los estudiantes de la carrera Tecnologías de la Información de la Universidad Estatal del Sur de Manabí tengan a la mano el ordenador correcto que proporcione el trabajo y mejore el rendimiento académico de los mismos.

Es así que el objetivo busca desarrollar las habilidades que permitan elegir correctamente un ordenador para brindar mayor comodidad al momento de gestionar las tareas que se ejecuten por parte del estudiante.

\section{CONCLUSIONES}

Pues bien, la computadora es un artefacto electrónico capaz de establecer, procesar y elegir deducciones como una información. En el mundo actual, con el manejo inmenso de conocimientos e información y la complejidad que presenta esta en el siglo XXI (época de crecimiento tecnológico), es imprescindible contar con una herramienta (dispositivos microinformáticos) que admita manejar información con eficiencia y flexibilidad.

Es indispensable conocer las partes del dispositivo microinformático tales como almacenamiento, memoria, procesador, periféricos de entrada y salida, entre otras que lo complementan., ya que de ellos depende el rendimiento que espera el estudiante al momento de ejecutar procesos o tareas. 
Jose E. Alava Cruzatty, Magdalena Toala Zambrano, Victor A. Figueroa Castillo, Genesis M. Rodriguez Sinisterra

Por último, se concluye que es importante tomar en cuenta los componentes y las capacidades que brinda un dispositivo microinformático al momento de adquirirlo.

También es indispensable saber la necesidad que el estudiante informático requiere ya que esta juega un papel importante al momento de darle uso de acuerdo a las necesidades del mismo.

Hay que tomar en cuenta lo siguiente: "Un ordenador para un empresario no es el mismo que para un estudiante informático, por lo que cada uno de ellos cumplen roles diferentes en sus profesiones.”

\section{REFERENCIAS}

Albornoz, M., Estébanez, M. E., \& Alfaraz, C. (Enero de 2005). Revista iberoamericana de ciencia tecnología y sociedad. (R. i. soc., Ed.) Obtenido de www.scielo.org.ar: http://www.scielo.org.ar/scielo.php?pid=S1850$00132005000100005 \&$ script=sci_arttext\&tlng=en

BENEFICIOS, T. L. (s.f.). TODOS LOS BENEFICIOS. Obtenido de beneficiosdel.net: https://beneficiosdel.net/la-tecnologia-en-la-sociedad/

Berral Montero , I. (2014). Manejo y Mantenimiento de Sistemas y Componentes Informaticos. Madrid,

España: Paraninfo. Obtenido de

https://books.google.com.ec/books?hl=es\&lr=lang_es\&id=Otv7CAAAQBAJ\&oi=fnd\&pg=PR13\&d

$\mathrm{q}=$ introducci\%C3\%B3n+a+los+equipos+microinform\%C3\%A1ticos, +estructura+externa+de+un+e quipo+microinform\%C3\%A1tico,+estructura+f\%C3\%ADsico-

funcional+de+un+equipo+microinform $\% \mathrm{C}$

Blogia. (27 de DIClEMBRE de 2004). Microinformatica. Obtenido de Microinformatica: https://microinformatica.blogia.com/2004/122604-estructura-externa-de-un-equipomicroinform-tico.php

Castillo T., J. L. (1 de Marzo de 2013). Solo Ciencia. Obtenido de www.solociencia.com: https://www.solociencia.com/informatica/computador-historia-historia.htm

Cosoi P., E. (2001). Cómo elegir un computador. Revista chilena de pediatría, 1.

Delfin, J. (19 de Febrero de 2013). historia del computador y sus caracteristicas. Obtenido de historacomputadorcaracteristicas.blogspot.com:

http://historacomputadorcaracteristicas.blogspot.com/

EL ESPECTADOR. (12 de Dlciembre de 2009). Computador según la necesidad. Diario EL ESPECTADOR.

Henry Bautista. (11 de Mayo de 2018). academia. Obtenido de academia: http://www.academia.edu/10366477/INTRODUCCI\%C3\%93N_A_LOS_EQUIPOS_MICROINFORM \%C3\%81TICOS

292 UNESUM-Ciencias. Publicación cuatrimestral. Vol. 4, No. 4 (Septiembre-Diciembre), Año 2020. 
Hernadez, V. (14 de Noviembre de 2016). GUIA: APRENDE A ARMAR TU PC . Obtenido de www.poderpda.com: https://www.poderpda.com/hardware/guia-elegir-mejor-fuente-de-poder$\mathrm{pc} /$

Montero, I. B. (2010). Operaciones auxiliares de montaje de componentes informaticos. Madrid, España: Paraninfo. https://books.google.com.ec/books?id=N9GgbaYTiylC\&printsec=frontcover\&hl=es\#v=onepage\& $q \& f=f a l s e$

Montero, I. B. (2016). Equipos Microinformaticos. Madrid, España: Paraninfo.

Pueyo, A. (18 de Noviembre de 2016). Tecnología y sociedad. Obtenido de sites.google.com: https://sites.google.com/a/correo.unimet.edu.ve/tecnologia-y-sociedad-influencia-del-uso-dela-tecnologia-en-el-comportamiento-de-la-sociedad/home/pagina-web-3

R. A. (6 de Enero de 2016). Computer Hoy. Obtenido de computerhoy.com: https://computerhoy.com/noticias/hardware/todo-que-necesitas-saber-memoria-ram-37541

R. A. (5 de Marzo de 2016). COMPUTER HOY. Obtenido de computerhoy.com/: https://computerhoy.com/noticias/zona-gaming/como-elegir-caja-sistemas-refrigeracion-tu-pc41325

Ruben Andres. (24 de Enero de 2016). Computer Hoy. Obtenido de computerhoy.com: https://computerhoy.com/noticias/hardware/todo-que-necesitas-saber-elegir-placa-base-36293

T., J. L. (s.f). Sólo Ciencia. Obtenido de Estas herramientas necesitan de una plataforma en la cual ejecutarse. Este es el papel del sistema operativo de una máquina computacional, que permite gestionar ficheros, llamadas al sistema, entre otras acciones. Siendo Linux un sistema operativo muy efi 